\title{
Breaching the mucosal barrier by stealth: an emerging pathogenic mechanism for enteroadherent bacterial pathogens
}

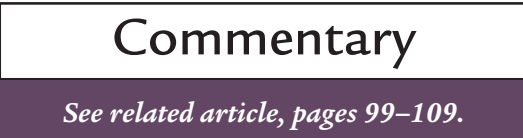

\author{
James M. Fleckenstein ${ }^{1}$ and Dennis J. Kopecko ${ }^{2}$ \\ ${ }^{1}$ Department of Medicine, University of Tennessee and Veterans Affairs Medical Center, Memphis, Tenessee 38104,USA \\ ${ }^{2}$ Laboratory of Enteric and Sexually Transmitted Diseases, Food and Drug Administration, Center for Biologics Evaluation and Research, \\ Bethesda, Maryland, USA \\ Address correspondence to: Dennis J. Kopecko, Laboratory of Enteric and Sexually Transmitted Diseases, Food and Drug Administration, \\ Center for Biologics Evaluation and Research, Bethesda, Maryland 20892, USA. \\ Phone: (301) 496-1893; Fax: (301) 480-5047; E-mail: kopecko@cber.fda.gov.
}

The clinical and molecular understanding of bacterial diseases of the gastrointestinal tract, developed largely over the past 50 years, has led to our current assignment of these causative agents into an evolving set of mechanistic categories (see Table 1). Early studies revealed that some organisms (Staphylococcus aureus among them) produce toxins in contaminated foods and that, upon ingestion, these preformed toxins trigger a rapid-onset, net jejunal secretion of electrolytes and water, typically lasting less than 24 hours. In these illnesses, the causative organism need not be present in the intestine. In contrast, the "enterotoxigenic" pathogens, typified by Vibrio cholerae and enterotoxigenic Escherichia coli (ETEC), are noninflammatory and must colonize the gastrointestinal tract in order to deliver toxins to the mucosa. These toxins typically effect cAMP- or cGMP-mediated net $\mathrm{Cl}^{-}$secretion, often resulting in a large-volume diarrhea. Recent studies of certain strains of Bacteroides fragilis indicate that these agents can serve as large bowel mediators of enterotoxigenic disease via a toxin that disrupts the mucosal barrier (1).

Another long-recognized grouping of enteric bacterial diseases is the "invasive" category, which can be subdivided based upon the extent of dissemination within the host. The most superficial invaders, Shigella spp. and enteroinvasive E. coli, have similar, if not identical, virulence properties. These organisms invade the epithelium of the colon and terminal ileum, spread intercellularly via actin-filament projections (2), and cause focal microulcerations of the ileocecal mucosa. The submucosal group is exemplified by Yersinia enterocolitica and Yersinia pseudotuberculosis, which transcytose across the mucosal epithelium and proceed to the regional lymph nodes, causing severe abdominal pain and, occasionally, mesenteric lymphadenitis. Recent clinical and experimental data suggest that Campylobacter jejuni is an invasive pathogen that can translocate across the mucosa, and that may survive submucosally, but does not typically reach the bloodstream (3). The third invasive subgroup is typified by certain Salmonella serovars (e.g., Typhi and Paratyphi) that invade and translocate across the epithelium. These organisms enter and apparently survive for many hours in submucosal monocytes, which may serve to transport these pathogens to distal sites in the host (e.g., spleen, liver, and bone marrow).

The characterization of a previously unrecognized group of pathogenic $E$. coli (i.e., enteropathogenic E. coli [EPEC], enteroaggregative E. coli [EAEC], diffusely adherent $E$. coli [DAEC], and enterohemmorhagic E. coli [EHEC]) since the 1950s has led to the establishment of the enteroadherent category of disease. These agents colonize the small

and/or large intestine, do not appear to be invasive to any appreciable extent in vivo, and, with the exception of shigalike toxins of EHEC, are not known to elaborate any of the classical enterotoxins, yet they cause diarrheal illness. Classical EPEC serotypes are known to induce "pedestal" or "cup-like" rearrangements, referred to as attaching and effacing lesions, of epithelial cell membranes. The attaching bacteria erode the microvilli via a rearrangement of cytoskeletal actin into host membrane pedestal structures that support the organism. EAEC and DAEC display characteristic, but different, adherence patterns on cultured intestinal cells and have not been shown to cause any striking membrane effacement. Although certain aspects of EPEC pathogenesis, such as adherence and the attaching and effacing phenotype, have been studied elegantly and in detail (4), the mechanisms by which these and other enteroadherent organisms induce inflammation and diarrhea have only recently started to emerge (5).

\section{Table 1}

Pathogenic mechanisms used by diarrheogenic bacteria
Category
Representative pathogens
I. Preformed enterotoxin
Staphylococcus aureus, Bacillus cereus
II. Enterotoxigenic, noninflammatory
A. Small bowel
B. Large bowel
ETEC, Vibrio cholerae
Toxigenic Bacteroides fragilis
III. Enteroadherent
A. Attaching and effacing
B. Noneffacing
EPEC, EHEC
EAEC, DAEC, S. Typhimurium
IV. Invasive
A. Superficial mucosal invasion Shigella spp., EIEC
B. Submucosal invasion
Yersinia enterocolitica, Campylobacter jejuni
C. Submucosal invasion and intrahost dissemination
Salmonella serovars; e.g., Typhi and Paratyphi
S. Typhimurium, Salmonella enterica serovar Typhimurium; ETEC, enterotoxigenic Escherichia coli; EPEC, enteropath- ogenic E. coli; EHEC, enterohemmorhagic E. coli; EAEC, enteroaggregative E. coli; DAEC, diffusely adherent E. coli; EIEC, enteroinvasive E. coli. 
Salmonella enterica serovar Typhimurium (now abbreviated $S$. Typhimurium) have previously been considered members of the invasive category, because of their invasiveness in cultured cells and their ability to cause a typhoid-like illness in mice. Indeed, $S$. Typhimurium may occasionally be isolated from extraintestinal sites in humans including the bloodstream, particularly in immunocompromised hosts. However, these pathogens are more typically associated in humans with diarrheal illness of mild to moderate severity accompanied by fever and the appearance of fecal leukocytes. Although $S$. Typhimurium may trigger limited invasion of the intestine, as may occur with other enteroadherent pathogens, mucosal invasion does not appear to be a prominent aspect of normal disease. As now shown in this issue of the JCI (6), S. Typhimurium appear to be prime examples of organisms that cause diarrheal disease through a general mechanism that may characterize many bacteria commonly placed in the enteroadherent category of intestinal diseases.

IL-8 as an inflammatory mediator

The migration of polymorphonuclear leukocytes (PMNs) into the intestinal mucosa is a hallmark of both inflammatory infectious diarrhea (7) and the idiopathic inflammatory bowel diseases
(IBDs) (8) for which chronic infection with an as-yet unidentified microorganism remains an attractive hypothesis. Groups interested in both of these clinical entities have naturally focused on a principal chemoattractant for PMNs, the chemokine IL-8.

Earlier work clearly demonstrated that a number of enteropathogens, including nontyphoidal Salmonella, induce target epithelial cells to produce this proinflammatory cytokine while causing only a mild gastroenteritis $(7,9)$. However, Shigella infection triggers IL-8 production, and the intense ensuing inflammatory response can lead to epithelial cell destruction and histopathologic lesions of the colon that are indistinguishable from acute ulcerative colitis (10). Other pathogens such as EAEC trigger IL-8 production, which results in more subtle, but no less significant, problems such as persistent diarrhea and growth impairment in children in developing countries (11). These observations suggest that IL- 8 stimulation may serve different purposes in different diseases and, likewise, that solicited PMNs may serve multiple roles in pathogenesis. During shigellosis, PMNs may primarily serve to stem the overt breach of the epithelium and to contain the organism. In contrast, in nontyphoidal salmonelloses the bacteria adhere to the intestine and initially trig- ger IL-8 production which recruits PMNs basolaterally, where they may prevent further spread of a few transcytosed bacteria. Subsequently, these basolateral PMNs can transmigrate, via bacteria-induced chemoattractants, to the luminal surface (12) where they may counter organisms at the gut surface and/or stimulate diarrhea.

\section{Induction of epithelial cell IL-8 production by bacterial flagellin}

Recently, in the JCI, Steiner et al. demonstrated that a unique bacterial flagellin protein produced by EAEC is a potent stimulus for IL-8 secretion in cultured epithelial cells (13). These authors also demonstrated that purified flagellin from selected EPEC and EHEC strains also stimulated IL-8 secretion by target cells. In the current issue of the JCI, Gewirtz and coworkers (6) have expanded upon their earlier studies showing that $S$. Typhimurium triggers basolateral IL-8 secretion from cultured model epithelia (14) via $\mathrm{Ca}^{++}$mediated activation of the NF- $\kappa B$ pathway. Previous work showed that $S$. Typhimurium only associates with a small proportion of model epithelial cells but that it induces a robust IL- 8 release, as if all cells were somehow responding. Gewirtz et al. hypothesized that $S$. Typhimurium might trigger epithelial exocytosis of a proin-

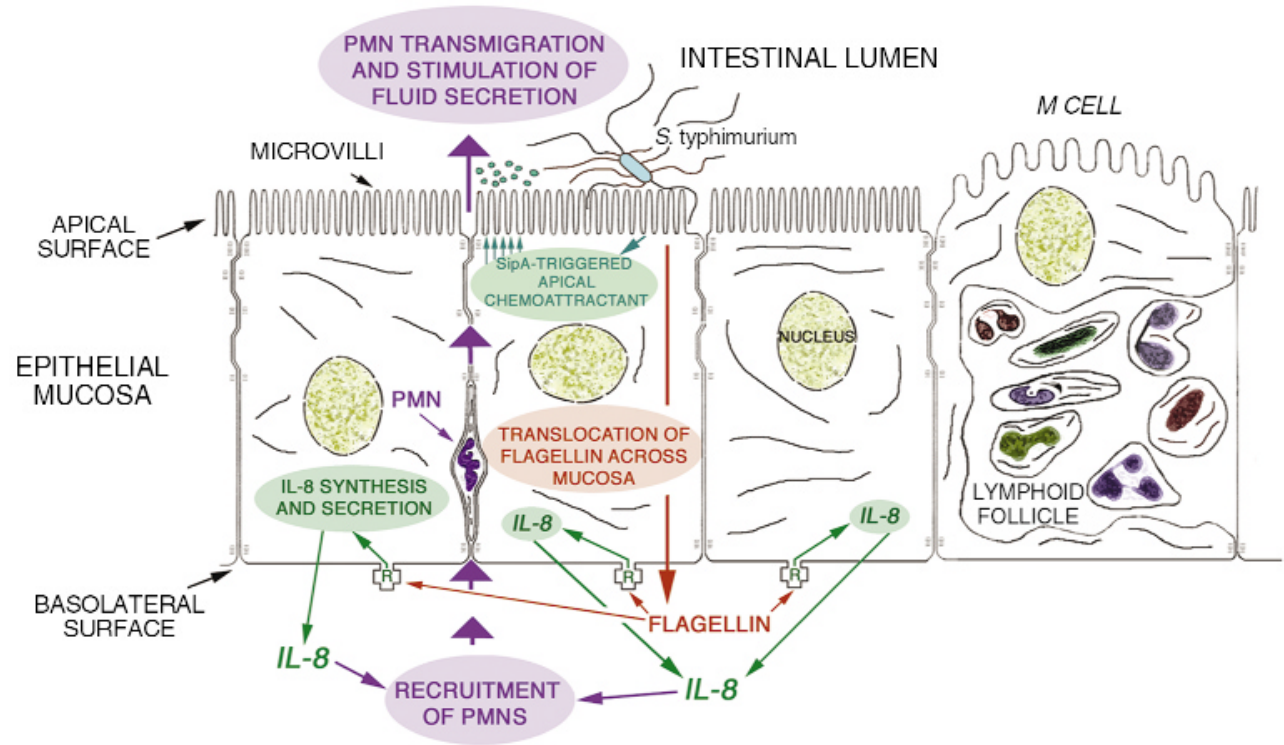

Figure 1

Working model for flagellin-induced stimulation of inflammatory diarrhea by S. Typhimurium. Schematic shows monolayer of intestinal epithelial cells with an adherent $S$. Typhimurium. Flagellin is translocated by an undetermined mechanism (depicted in red) to the basolateral surface, where it interacts with putative receptors (R) that trigger IL-8 production (depicted in dark green) and basolateral recruitment of PMNs. S. Typhimurium has recently been reported to stimulate production of an apical chemoattractant (depicted in light green) that aids in PMN transmigration to the luminal surface, where these leukocytes trigger fluid secretion (pathway depicted in blue). 
flammatory mediator that might activate IL-8 synthesis via a mechanism similar to TNF- $\alpha$ (6).

Indeed, these researchers found a soluble mediator, present after 1 hour in the basolateral fluids of monolayer cells infected apically with $S$. Typhimurium. This soluble mediator could trigger IL-8 synthesis when applied basolaterally, but not apically, to fresh model epithelial cells. Further study revealed that the IL-8 mediator was not of eukaryotic origin, but rather represented bacterial flagellin, and that supernatants of flagellated, but not of nonflagellated mutants of, S. Typhimurium cultures could induce IL-8 synthesis when applied basolaterally to model epithelia. Moreover, the culture supernatants of five out of seven strains of flagellated, but nonpathogenic, commensal E. coli induced IL-8 in this basolateral exposure system. Purified flagellin, added apically in the absence of bacteria, was unable to translocate across model epithelia or to elicit IL-8 secretion, emphasizing the basolateral localization of this activation. As one might expect, flagellin translocation across model epithelia was not mediated by flagellated strains of commensal E. coli. Limited studies of $S$. Typhimurium mutants indicate that type III secretory-mediated interactions of bacteria with host cells are not essential for flagellin translocation. While the mechanism of flagellin translocation remains undefined, the ability to translocate flagellin across the mucosa appears to be an important determinant of virulence in certain bacteria that elicit IL-8 in the absence of a physical break in the mucosa (see Figure 1).

Flagellin purified from wild-type $S$. Typhimurium induced detectable IL-8 secretion at concentrations below 1 $\mathrm{ng} / \mathrm{ml}$, more potent than the proinflammatory agonist TNF- $\alpha$. Although LPS is a key activator of subepithelial macrophages, flagellin now appears to play a major role in triggering the intestinal mucosal stimulation of an inflammatory response.

\section{Current concepts, uncertainties, and implications}

Figure 1 depicts the events proposed to occur during $S$. Typhimurium-induced stimulation of inflammatory diarrhea. In this working model, translocation of flagellin to the basolateral surface is required to stimulate the production of IL-8, resulting in PMN recruitment from the lamina propria. The mechanism of flagellin translocation is unclear. The recent demonstration of flagella-dependent secretion of YplA, a virulence protein of Y. enterocolitica (15), raises the intriguing possibility that flagellin could be delivered to the cell by the very same structure that it helps to form. As suggested by recent studies with $S$. Typhimurium, additional bacterial virulence factors induce the target epithelial cells to secrete neutrophil chemoattractants at the apical surface, which in turn direct the transmigration of basolateral PMNs to the intestinal lumen $(12,16)$. Lee et al. recently demonstrated that SipA, a substrate of the type III secretion system located on a Salmonella pathogenicity island (SPI1), provides an essential stimulus by activating a protein kinase $\mathrm{C}$-dependent signal transduction pathway to modulate the polarized production of apical chemoattractants (12) (Figure 1). Presumably, the directed migration of neutrophils to the luminal surface is important to the induction of diarrhea, as first proposed by Giannella et al. (17). Studies by Madara et al. (18) have suggested that PMNs stimulate epithelial chloride secretion by delivery of a neutrophil-derived secretogogue, 5' AMP, to the apical surface of epithelial cells. Conversion of $5^{\prime}$-AMP by apical membrane nucleotidase provides adenosine that targets local receptors, which ultimately signal chloride secretion. More recent studies have suggested that in addition to recruiting leukocytes, Salmonella may perturb multiple epithelial-cell processes that normally govern chloride secretion, including prostaglandin formation (19) and inositol-based signal pathways (20). Moreover, Salmonella and other pathogens have recently been shown to induce upregulation of intestinal galanin receptors, which upon activation lead to chloride secretion (21). Hence, induction of diarrhea by $S$. Typhimurium might be viewed as a highly orchestrated series of events involving both the migration of PMNs and direct stimulation of chloride secretion by alteration of cellular homeostatic mechanisms.

It remains to be seen whether all bacteria categorized as enteroadherent (Table 1) employ comparable strategies, or whether delivery of flagellin to the basolateral surface represents a common thread among otherwise disparate tactics of diarrheogenesis. Likewise, the recent identification of a novel putative enterotoxin of EAEC (5) serves as a reminder that pathogenesis is generally complex and multicomponent. Thus, recruitment of neutrophils may be only one of several diarrheogenic pathways among this group of bacteria. Translocation of flagellin certainly does not represent the only mechanism by which bacteria recruit neutrophils. For example, it is known that the cag pathogenicity island of Helicobacter pylori is required for induction of IL-8 by gastric epithelium $(22,23)$. Similarly, recent studies have suggested that cytolethal distending toxin (24) is a primary stimulus for IL-8 secretion induced by $C$. jejuni. It is also unclear whether Shigella, which have only recently been shown to produce functional flagella (25), use flagellin as an effector molecule in the recruitment of PMNs.

Despite these gaps in our understanding of pathogenesis, the studies outlined here have broad and significant implications in unraveling the molecular pathogenesis of diseases caused by nontyphoidal salmonellae and, perhaps, other enteroadherent diarrheal pathogens. Likewise, Gewirtz and coworkers (6) have opened new avenues to explore the pathogenesis of IBD. Whether IBD represents the consequence of repeated exposure to ubiquitous flagellin from nonpathogenic organisms through incidental or host-induced breaks in the epithelial barrier, or whether these diseases are contingent on an orchestrated breach of the epithelium directed by a pathogen, is likely to remain the focus of intense investigation.

\section{Acknowledgments}

We thank Richard Kenney, Stephen Savarino, and Gerald Keusch for their critical reading of this Commentary, and James McDaniel for work on the illustration.

\footnotetext{
1. Wu, S., Lim, K.C., Huang, J., Saidi, R.F., and Sears, C.L. 1998. Bacteroides fragilis enterotoxin cleaves the zonula adherens protein, E-cadherin. Proc. Natl. Acad. Sci. USA. 95:14979-14984.

2. Sansonetti, P.J., Tran Van Nhieu, G., and Egile, C. 1999. Rupture of the intestinal epithelial barrier and mucosal invasion by Shigella flexneri. Clin. Infect. Dis. 28:466-475.

3. Hu, L., and Kopecko, D.J. 2000. Interactions of Campylobacter with eukaryotic cells: gut luminal colonization and mucosal invasion mechanisms. In Campylobacter. 2nd edition. I. Nachamkin and M.J. Blaser, editors. American Society for Microbiology. Washington, DC, USA. 191-215.

4. Donnenberg, M.S., Kaper, J.B., and Finlay, B.B. 1997. Interactions between enteropathogenic Escherichia coli and host epithelial cells. Trends
} 
Microbiol. 5:109-114.

5. Eslava, C., et al. 1998. Pet, an autotransporter enterotoxin from enteroaggregative Escherichia coli. Infect. Immun. 66:3155-3163.

6. Gewirtz, A.T., et al. 2001. Salmonella typhimurium flagellin translocated across intestinal epithelial cells induces a proinflammatory response. J. Clin. Invest. 107:99-109.

7. Eckmann, L., Kagnoff, M.F., and Fierer, J. 1993. Epithelial cells secrete the chemokine interleukin-8 in response to bacterial entry. Infect. Immun. 61:4569-4574.

8. Daig, R., et al. 1996. Increased interleukin 8 expression in the colon mucosa of patients with inflammatory bowel disease. Gut. 38:216-222.

9. Jung, H.C., et al. 1995. A distinct array of proinflammatory cytokines is expressed in human colon epithelial cells in response to bacterial invasion. J. Clin. Invest. 95:55-65.

10. Sansonetti, P.J., Arondel, J., Huerre, M., Harada, A., and Matsushima, K. 1999. Interleukin- 8 controls bacterial transepithelial translocation at the cost of epithelial destruction in experimental shigellosis. Infect Immun. 67:1471-1480.

11. Steiner, T.S., Lima, A.A., Nataro, J.P., and Guerrant, R.L. 1998. Enteroaggregative Escherichia coli produce intestinal inflammation and growth impairment and cause interleukin-8 release from intestinal epithelial cells. J. Infect. Dis. 177:88-96.

12. Lee, C.A., et al. 2000. A secreted Salmonella protein induces a proinflammatory response in epithelial cells, which promotes neutrophil migration. Proc. Natl. Acad. Sci. USA. 97:12283-12288.

13. Steiner, T.S., Nataro, J.P., Poteet-Smith, C.E., Smith, J.A., and Guerrant, RL. 2000. Enteroaggregative Escherichia coli expresses a novel flagellin that causes IL-8 release from intestinal epithelial cells. J. Clin. Invest. 105:1769-1777.

14. Gewirtz, A.T., et al. 2000. Salmonella typhimurium induces epithelial IL-8 expression via $\mathrm{Ca}^{2+}$-mediated activation of the NF- $\mathrm{\kappa B}$ pathway. J. Clin. Invest. 105:79-92.

15. Young, G.M., Schmiel, D.H., and Miller, V.L. 1999. A new pathway for the secretion of virulence factors by bacteria: the flagellar export apparatus functions as a protein-secretion system. Proc. Natl. Acad. Sci. USA. 96:6456-6461.

16. McCormick, B.A., Parkos, C.A., Colgan, S.P., Carnes, D.K., and Madara, J.L. 1998. Apical secretion of a pathogen-elicited epithelial chemoattractant activity in response to surface colonization of intestinal epithelia by Salmonella typhimurium. J. Immunol. 160:455-466.

17. Giannella, R.A., Formal, S.B., Dammin, G.J., and Collins, H. 1973. Pathogenesis of salmonellosis. Studies of fluid secretion, mucosal invasion, and morphologic reaction in the rabbit ileum. J. Clin. Invest. 52:441-453.

18. Madara, J.L., et al. 1993. 5'-adenosine monophosphate is the neutrophil-derived paracrine factor that elicits chloride secretion from T84 intestin- al epithelial cell monolayers. I. Clin. Invest. 91:2320-2325.

19. Eckmann, L., et al. 1997. Role of intestinal epithelial cells in the host secretory response to infection by invasive bacteria. Bacterial entry induces epithelial prostaglandin h synthase- 2 expression and prostaglandin E2 and F2alpha production. J. Clin. Invest. 100:296-309.

20. Eckmann, L., et al. 1997. D-myo-Inositol 1,4,5,6tetrakisphosphate produced in human intestinal epithelial cells in response to Salmonella invasion inhibits phosphoinositide 3-kinase signaling pathways. Proc. Natl. Acad. Sci. USA. 94:14456-14460.

21. Matkowskyj, K.A., et al. 2000. Galanin-1 receptor up-regulation mediates the excess colonic fluid production caused by infection with enteric pathogens. Nat. Med. 6:1048-1051.

22. Censini, S., et al. 1996. cag, a pathogenicity island of Helicobacter pylori, encodes type I-specific and disease-associated virulence factors. Proc. Natl. Acad. Sci. USA. 93:14648-14653.

23. Segal, E.D., Lange, C., Covacci, A., Tompkins, L.S., and Falkow, S. 1997. Induction of host signal transduction pathways by Helicobacter pylori. Proc. Natl. Acad. Sci. USA. 94:7595-7599.

24. Hickey, T.E., et al. 2000. Campylobacter jejuni cytolethal distending toxin mediates release of interleukin-8 from intestinal epithelial cells. Infect. Immun. 68:6535-6541.

25. Giron, J.A. 1995. Expression of flagella and motility by Shigella. Mol. Microbiol. 18:63-75. 